doi: 10.32620/oikit.2018.81.08

УДК 629.7 .03

И.И. Редин, М.А. Шевченко

\title{
Улучшение топливной эффективности газотурбинного двигателя установкой в компрессоре надроторного устройства
}

\author{
Национальный аэрокосмический университет им. Н. Е. Жуковского \\ "Харьковский авиационный институт», Украина
}

\begin{abstract}
Представлен анализ влияния кольцевых канавок в наружной стенке корпуса осевого компрессора на течение в проточной части рабочего колеса и характеристики осевого компрессора, а также на топливную эффективность ГТД в целом. Изложен предположительный механизм воздействия течения в полости кольцевой канавки на основное течение в концевой области проточной части рабочего колеса осевого компрессора. Эффективность надроторного устройства в виде одиночной кольцевой канавки, ширина которой составляет 20 \% осевой проекции хорды концевого сечения лопатки рабочего колеса, проверена экспериментально в системе одноступенчатого и многоступенчатого осевого компрессора. Рост КПД компрессора с десятью одиночными кольцевыми канавками, установленными над входными кромками лопаток рабочих колес каждой ступени, обеспечил снижение удельного расхода топлива серийного ГТД на основных режимах его работы.
\end{abstract}

Ключевые слова: надроторное устройство, топливная эффективность, течение в радиальном зазоре, газодинамическая устойчивость, осевой компрессор.

\section{Введение}

Снижение удельного расхода топлива газотурбинного двигателя (ГТД) - актуальная задача всего исторического периода использования его в авиации, судовых и стационарных энергетических установках. Повышение КПД компрессора один из самых действенных способов повышения топливной эффективности ГТД.

Экспериментальные исследования в ХАИ [1, 2] показали, что конструктивно простые и технологичные надроторные устройства (НРУ) в виде совокупности кольцевых канавок (КК) в корпусе осевого компрессора (ОК) позволяют увеличить его КПД. Такой результат получен при оптимальном сочетании конструкции НРУ и его положения относительно лопаток рабочего колеса (РК).

На всех эксплуатационных режимах работы ГТД необходимо обеспечивать допустимые запасы газодинамической устойчивости (ГДУ). Одним из путей решения этой задачи также является применение НPУ [3 - 5].

\section{1 Постановка проблемы и анализ публикаций}

В литературе рассматривают различные HРУ [2,6 -13], которые можно разделить на две характерные группы:

1. Надроторное устройство в виде дискретных щелей в стенке корпуса с присоединённым объемом, в полостях которого формируется пристеночное обратное течение вследствие продольного градиента давления (от выхода из рабочего колеса ко входу в него) [7 - 10].

2. Надроторное устройство в виде одной или нескольких кольцевых канавок («лабиринтного типа») в стенке корпуса компрессора с полостями, расположенными перпендикулярно оси вращения ротора, которые препятствуют формированию продольного обратного течения [2,7,11 - 13]. 
В первом случае обеспечивают большее увеличение запаса устойчивости при существенной потере эфрфективности [8,14,15]. Во втором случае использование КК приводит не только к меньшему эфффекту увеличения запаса ГДУ, но и к меньшим потерям эффективности [16]. В работах [1, 12] экспериментально установлено оптимальное положение КК в области входной кромки концевого сечения пера лопатки РК, обеспечивающее повышение КПД рабочего колеса.

В работе [17] показано, что КК над входной кромкой также стабилизирует режим слабого (не приводящего к разрыву характеристики ОК) частичного вращающего срыва и таким образом позволяет диагностировать предпомпажное состояние осевого компрессора.

В работах $[17,18]$ установлено, что применение надроторного устройства (HРУ) в виде кольцевой канавки позволяет расширить диапазон устойчивых режимов по расходу, характеризуемых коэфффициентом расхода $\overline{C_{a}}=\frac{C_{a}}{U_{\kappa}}$. При этом в работе [18] показано, что если напорная характеристика рабочего колеса имеет левую ветвь (положительный градиент $\frac{d p}{d C_{a}} \geq 0$ ), то максимальный эффрект по запасу ГДУ будет обеспечен при расположении КК около 50\% хорды. Однако если напорная характеристика РК имеет только правую ветвь (отрицательный градиент $\frac{d p}{d C_{a}}<0$ ), то КК эффрективна над входной кромкой концевого сечения пера лопатки РК $[1,12,19]$.

Кроме того, в работе [18] показано, что на оптимальном режиме по расходу $\bar{C}_{a_{\text {оnт }}}$, который соответствует максимальному значению КПД $\eta_{\max }$, при оптимальной глубине КК, его значение (в случае расположения КК над входной кромкой) практически не изменилось по сравнению с гладкой проточной частью (ГПЧ) (рис. 1).

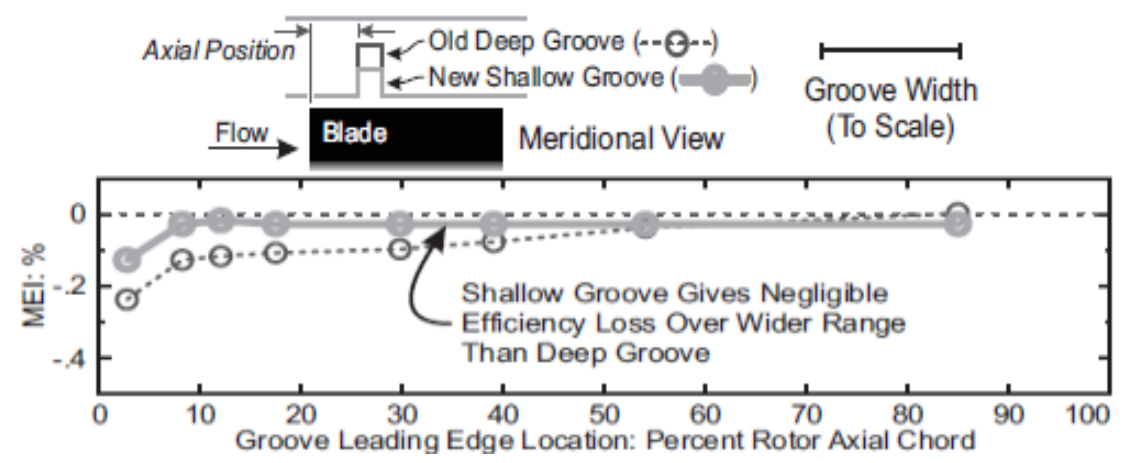

Рис. 1. Изменение КПД, вызванное КК различной глубины при ее перемещении вдоль хорды [18]

Как отмечалось ранее авторами работ [1, 12], в диапазоне режимов по рас-

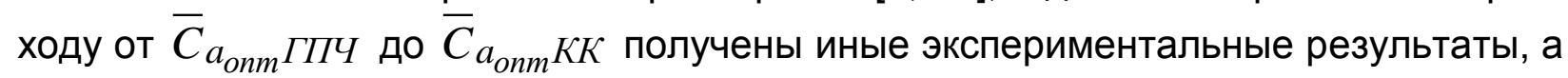
именно то, что КК над передней частью концевого профиля лопатки РК обеспечивает повышение КПД. В работе [12] показано, что расположение канавок вдоль 
всей проекции хорды и, особенно, в хвостовой ее части приносит меньший эффрект (рис. 2).

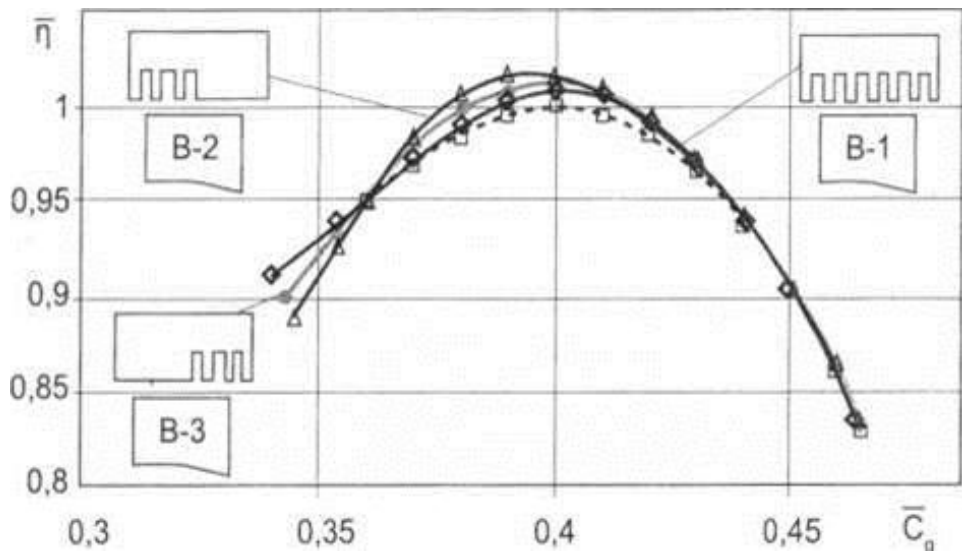

Рис. 2. Характеристики ступени при различных вариантах исполнения корпуса [12]:

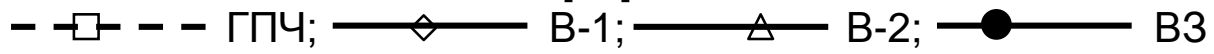

Опубликованные экспериментальные и расчетные данные демонстрируют отсутствие единой точки зрения по влиянию КК на течение в проточной части РК и характеристики ОК. Таким образом, актуальна задача выработки единого подхода в моделировании процесса воздействия КК на основной поток путем проведения дальнейших расчетных и экспериментальных исследований.

Целями данной работы являются:

- установление механизма влияния КК, расположенной в области входной кромки концевого сечения пера лопатки РК в корпусе ОК, на его характеристики и ГДУ;

- определение влияния КК, расположенных в корпусе МОК в области входных кромок концевых сечений пера лопатки РК, на характеристики ГТД и его топливную эфффективность.

\section{2 Механизм влияния КК в корпусе одноступенчатого компрессора на его характеристики и ГДУ}

В работах [20, 21] представлены результаты трехмерного экспериментального исследования полей течения в окрестности радиального зазора над лопатками рабочего колеса на расчетном режиме по расходу. Авторы показывают наличие скребкового эффекта со стороны давления концевого профиля пера лопатки и его влияния на картину течения в межлопаточном канале РК.

Подчеркнуто, что радиальные скорости внутри канала, направленные к периферии, незначительны вблизи входной кромки профиля, имеют максимальные значения при $z=0,25$ и при z $=0,979$ вблизи конца лопатки $(\bar{R}=0,89)$. Тангенциальное перемещение струи перетекания через радиальный зазор усиливается движением самой лопатки относительно корпуса. Струя имеет тенденцию к сворачиванию с образованием вихря. Происходит сложное перемешивание и вязкостное взаимодействие потока, перетекающего через зазор с пограничным слоем на кольцевой стенке. Эта область взаимодействия является областью максимальных потерь, в которой происходит интенсивное перемешивание потоков, приводящее к сильным завихрениям и рассеянию энергии. Область растет от 
входа в межлопаточный канал к его выходу. Тангенциальные составляющие относительной скорости быстро изменяются при переходе от конца лопатки $(\bar{R}=0,98)$ к кольцевой стенке. В области концов лопаток возникают большие градиенты тангенциальной составляющей абсолютной скорости потока, что является подтверждением заметного эффректа соскабливания [21]. Сделано заключение, что перетекание через радиальный зазор начинается в конце первой четверти хорды лопатки, причем максимальные значения расхода достигаются на середине хорды. Перетекание, происходящее в форме струи, внутри межлопаточного канала усиливается под действием вращения лопатки и струя распространяется внутрь канала от поверхности разряжения глубже, чем это наблюдается в неподвижных кольцевых решетках лопаток и в плоских решетках.

В работе [18] проведено обширное численное трехмерное исследование влияния НРУ на течение в окрестности полости КК на нерасчетном режиме работы ОК по расходу. Расчетная модель была верифицирована по результатам физического эксперимента. При положении канавки, определяемом параметром $\bar{\varepsilon}=8 \%$ (отношение абсолютного значения $\varepsilon$ к осевой проекции хорды концевого сечения пера лопатки $b_{a}$ ), поток в ней оказывает существенное влияние на основной поток проточной части РК. Радиальный поток внутри полости КК, а также в радиальном зазоре между торцом пера лопатки и ограничивающей стенкой корпуса уменьшает нагруженность профиля в концевой части пера лопатки в окрестности канавки. Проанализировано изменение компонент скорости потока по сравнению с ГПЧ. Осевая составляющая скорости потока вдоль оси вихря перетекания на стороне разрежения профиля значительно возрастает, при этом тангенциальная составляющая скорости потока в полости КК возрастает как со стороны разрежения, так и со стороны давления (как результат проявления вязкости рабочего тела). Радиальная составляющая скорости уменьшается со стороны разрежения и увеличивается со стороны давления, демонстрируя снижение интенсивности перетекания потока через концевое сечение пера лопатки под полостью кольцевой канавки (рис. 3).

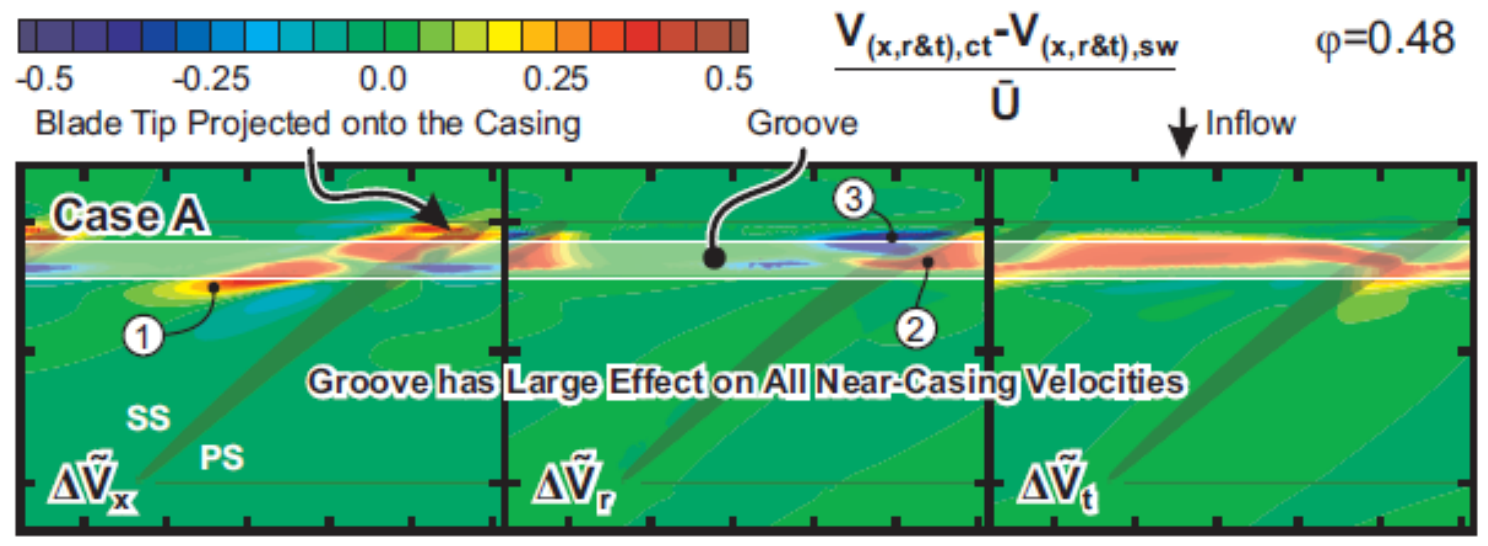

Рис. 3. Нормализованное изменение (по сравнению с ГПЧ) компонент абсолютной скорости потока, вызванное КК (расстояние от стенки корпуса ОК составляет 5\% от величины радиального зазора) [18]

Это согласуется с результатами работы [17], в которой обнаружено появление тангенциального течения в полости КК, интенсивность которого возрастала по мере уменьшения массового расхода через РК. 
Механизм возникновения тангенциального течения в полости КК обусловлен воздействием струй обратного течения от выхода ко входу в РК в области режимов по расходу, меньших оптимального. Резкое увеличение скорости тангенциального течения в полости КК наблюдается на режимах между границами устойчивой работы исходной ступени (ГПЧ) и ступени с КК. Скачок обусловлен образованием слабого частичного вращающего срыва (не приводящего к разрыву характеристики ОК) с противотоком в периферийной зоне РК, появление которого обнаружено при измерении пульсаций давления в полости канавки [17].

Косвенно это подтверждают результаты работы [18], где показано, что загруженность концевых сечений пера лопатки уменьшается (рис. 4).

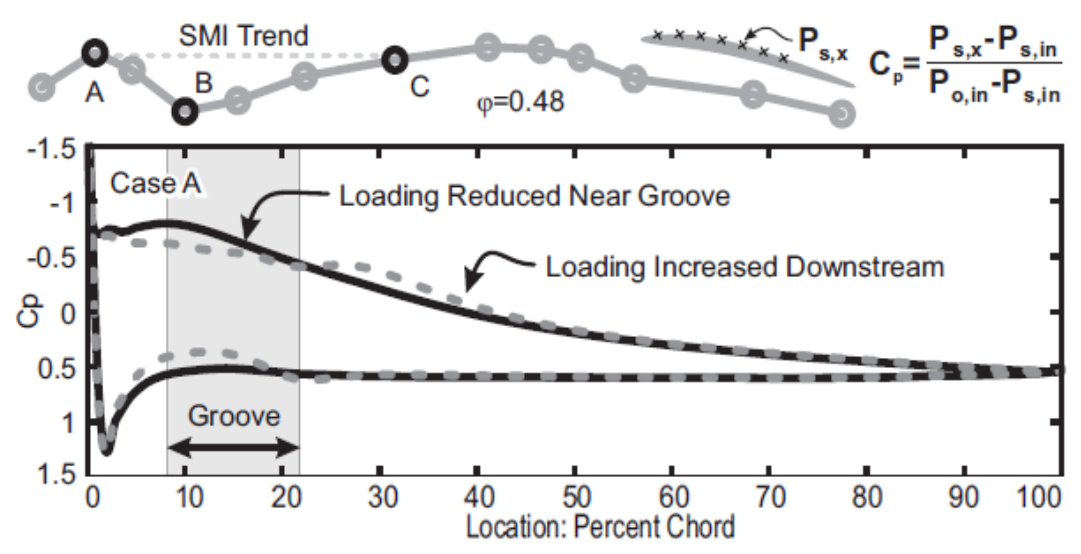

Рис. 4. Влияние КК на распределение давления вдоль профиля концевого сечения пера лопатки РК на расстоянии 2\% от стенки корпуса ОК [18]

Начало вихря перетекания перемещается к выходу по воздействием КК (рис. 5). Верификация модели проведена на 50 \% осевой проекции хорды и отображена в левой части рисунка.

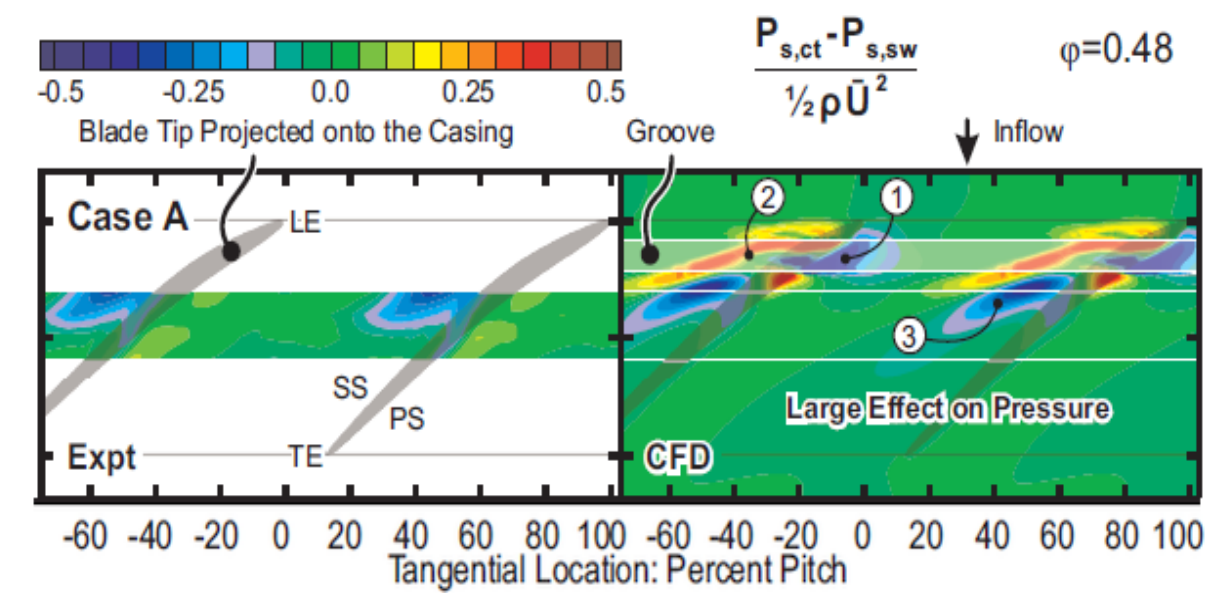

Рис. 5. Нормализованное изменение относительного статического давления, вызванное КК, на расстоянии от стенки корпуса ОК, равном 5\% радиального зазора [18]

Так как начало вихря сместилось вниз по потоку, вихрь расположен ближе к поверхности разрежения пера лопатки с установленной КК (рис. 6), что привело к снижению потерь в пристеночной области. 


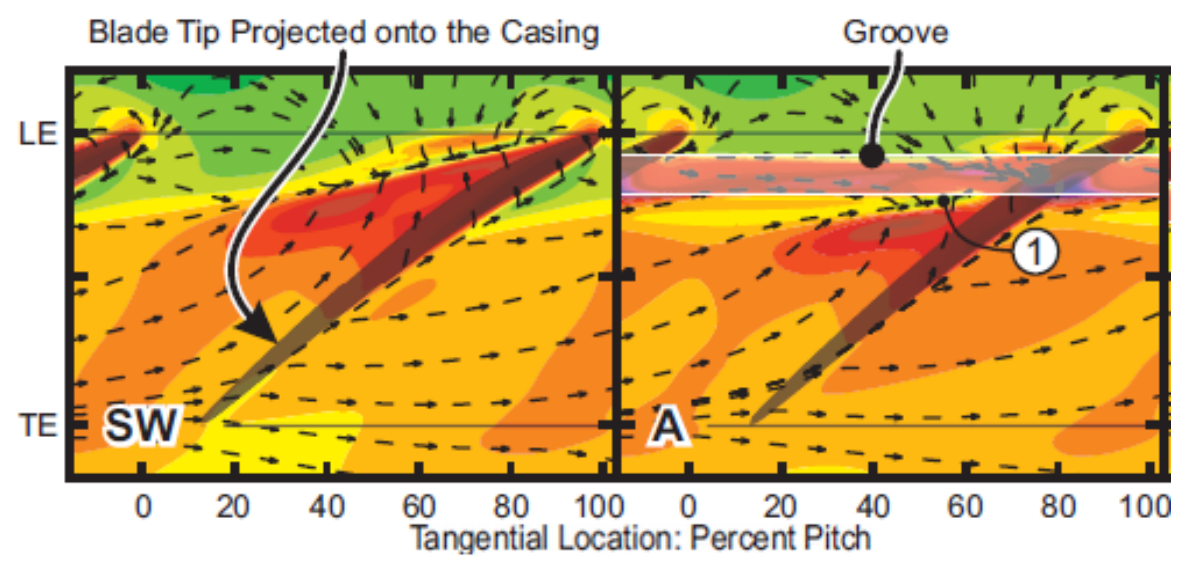

Рис. 6. Контурное и векторное распределение абсолютной скорости на расстоянии $5 \%$ радиального зазора от стенки корпуса ОК (в варианте стенки корпуса с ГПЧ и KK) [18]

Существенное влияние КК заключается в том, что полость КК для основного потока в проточной части РК - это резкое местное увеличение площади поперечного сечения проточной части (местный скачок дифффузорности течения под полостью КК), дополнительные потери полного давления. Интенсивность перетекания на стенке корпуса над РК (с выхода на вход в РК) уменьшается.

В работе [22] проведено экспериментальное исследование влияния величины присоединённого объема на границу устойчивых режимов и напорную характеристику РК. Установлено, что варьирование положения, КК и величины присоединенного объема практически не меняет исходную напорную характеристику рабочего колеса. Кроме того, изменение положения КК относительно лопаток РК позволило определить наиболее эфффективный вариант установки - КК над входной кромкой лопатки РК (рис. 7, а).
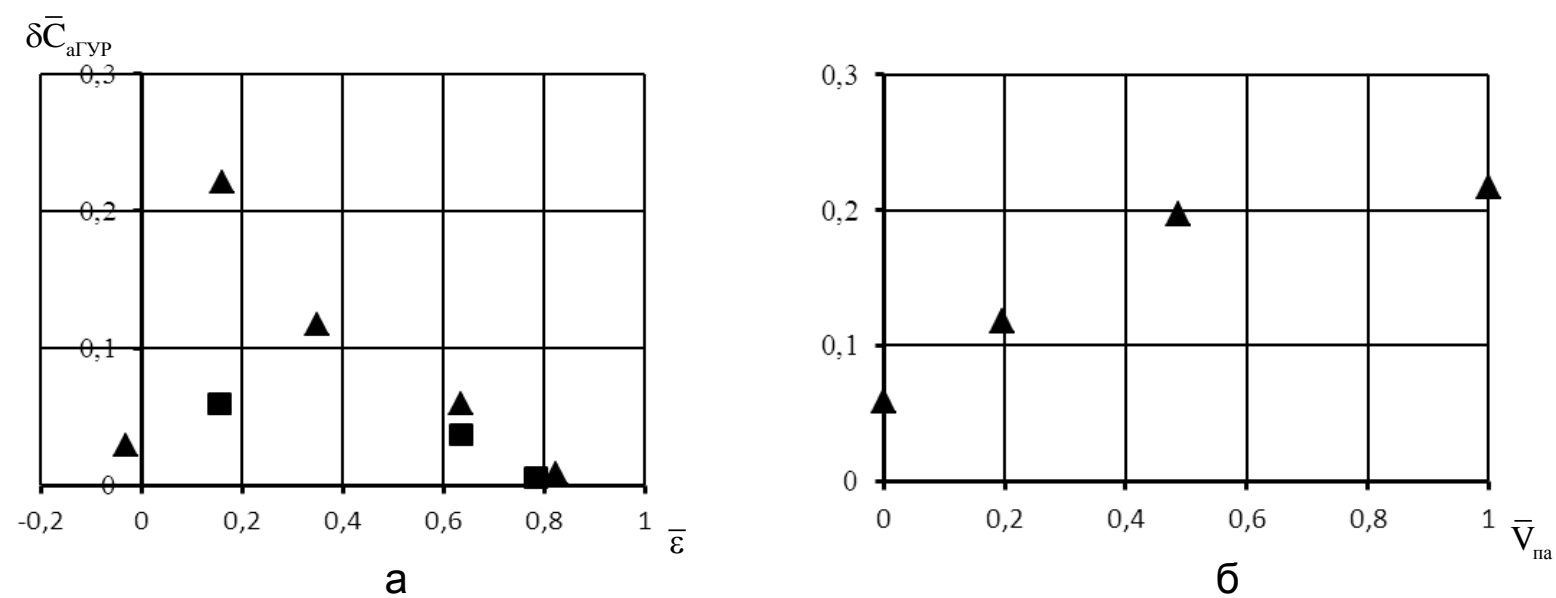

Рис. 7. Влияние на границу устойчивых режимов положения КК и величины присоединенного объема:

$$
\text { a }-\boldsymbol{\Delta}-\overline{\boldsymbol{V}}_{\boldsymbol{\Pi} \boldsymbol{O}}=1 ; \boldsymbol{\square}-\overline{\boldsymbol{V}}_{\boldsymbol{\Pi} \boldsymbol{O}}=0 ; \sigma-\bar{\varepsilon}=0,1
$$

Эффрективность работы устройства по смещению границы устойчивых режимов оценивалась параметром 


$$
\delta \bar{C}_{a_{\Gamma У P}}=\frac{\bar{C}_{a_{\Gamma У P}}^{*}-\bar{C}_{a_{\Gamma У P}}}{\bar{C}_{a_{\Gamma У P}}},
$$

где $\bar{C}_{a_{\Gamma у P}^{*}}^{*}$ - граница устойчивых режимов работы в исходном варианте (ГПЧ).

Из рис. 3, б следует, что эффективность устройства существенно зависит и от величины присоединенного объема, характеризуемой параметром $\bar{V}_{\Pi О}=V_{\Pi О} / V_{u c x}$, где $V_{u c x}$ - исходное (наибольшее) значение, выбранное из конструктивных соображений. Характер изменения $\delta \bar{C}_{a_{Г У Р}}$ на этом рисунке указывает, что увеличение присоединенного объема целесообразно до определенной величины, при превышении которой эффективность КК по смещению границы устойчивых режимов практически не меняется [22].

Полученные результаты дополняют сделанный в работе [18] вывод о том, что более глубокие канавки обеспечивают более высокий запас устойчивости, но снижают эффективность по сравнению с неглубокими канавками.

Экспериментальные исследования на ступени с большим диаметром втулки, геометрические параметры которой приведены в работе [17], подтвердили положительное влияние такой кольцевой канавки на КПД рабочего колеса и границу устойчивых режимов (ГУР) (рис. 8).

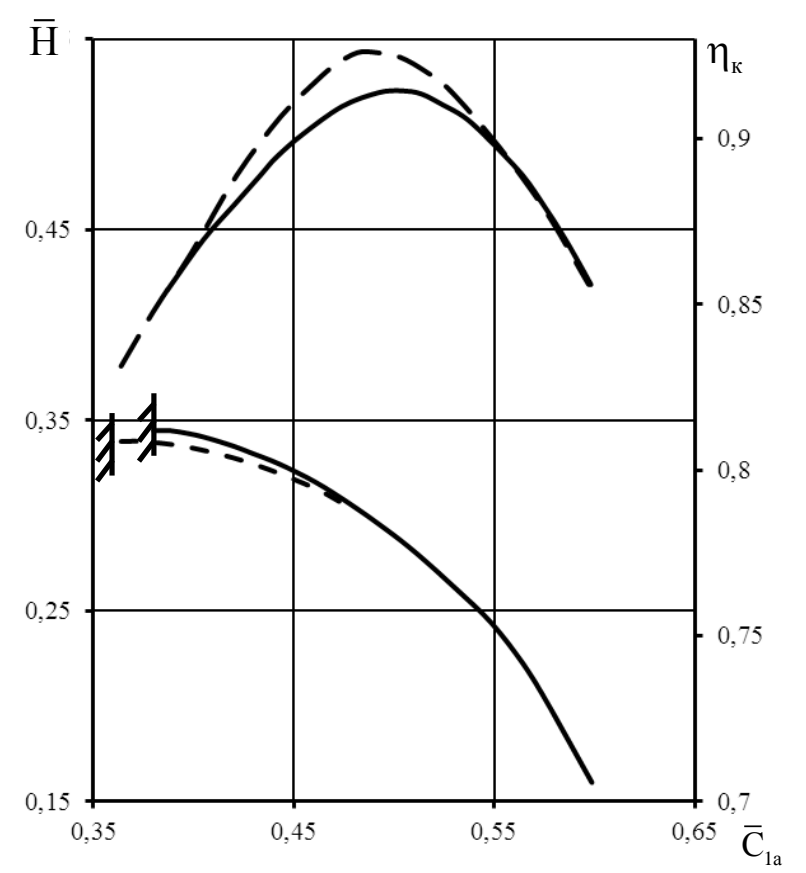

Рис. 8. Характеристика рабочего колеса одноступенчатого осевого компрессора:

$$
\begin{gathered}
- \text { исходный компрессор с гладкой проточной частью (ГПЧ); } \\
\text {----- - компрессор с кольцевой канавкой в корпусе над входными } \\
\text { кромками лопаток рабочего колеса; } \\
\text { - граница газодинамической устойчивости компрессора }
\end{gathered}
$$

Максимальное значение КПД возросло на 1,7\%, а соответствующий ему ко-

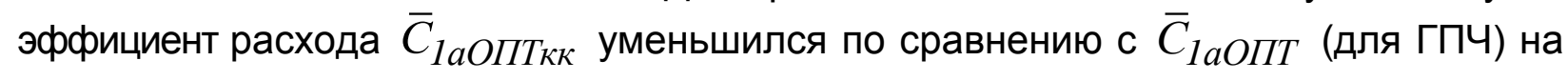


2\%. В целом диапазон режимов по расходу, в котором получено повышение КПД, соответствует диапазону режимов повышенной нагруженности венца рабочего колеса (от оптимального до ГУР). В этом диапазоне режимов действительный напор по мере приближения к ГУР снижается все в большей степени по отношению к напору исходной ступени. Граница газодинамической устойчивости сместилась в сторону меньших расходов на 3,5\% [1]. Изображенная на рис. 8 характеристика рабочего колеса получена по методике, описанной в работе [23].

Оптимальный режим по характеристике компрессора с КК (рис. 8) сместился в сторону меньших расходов по сравнению с ГПЧ. Это свидетельствует об уменьшении суммарных потерь в межлопаточном канале РК при установке КК, которое обусловлено смещением точки отрыва вихря на стороне разряжения вниз по потоку (рис. 6).

Из рис. 8 видно, что по сравнению с рис. 1 получен прирост КПД.

\section{2 Влияние КК над входными кромками рабочих лопаток в системе многоступенчатого осевого компрессора на его характеристики и ГДУ}

Эффрективность кольцевой канавки над входными кромками рабочих лопаток в системе многоступенчатого осевого компрессора (МОК) проверена на десятиступенчатом осевом компрессоре. Испытание проведено на стенде РД-02 по стандартной методике [24]. Сняты характеристики, определена граница газодинамической устойчивости (рис. 9). Исследования выполнены на режиме по частоте вращения ротора $\bar{n}_{n p}=0,96$, на котором компрессор имеет максимальное значение КПД.

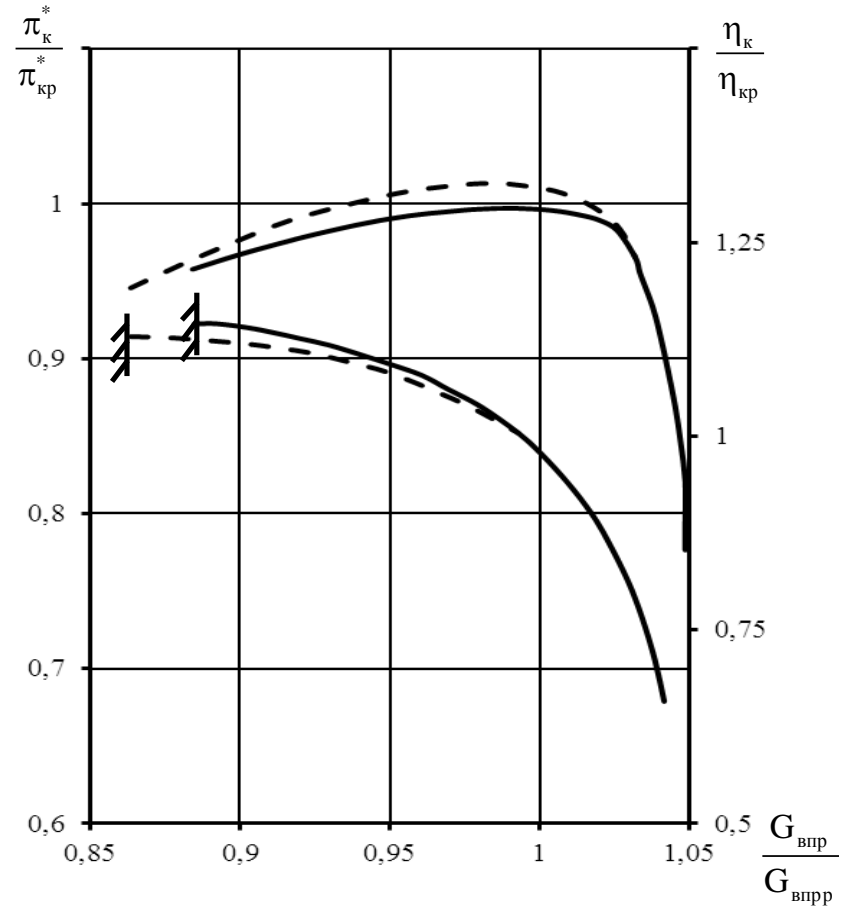

Рис. 9. Характеристика десятиступенчатого осевого компрессора:

- исходный компрессор с гладкой проточной частью;

-------- - компрессор с кольцевой канавкой в корпусе над входными кромками лопаток рабочего колеса;

才 - граница газодинамической устойчивости компрессора 
Анализ изменения опытных характеристик МОК показал качественную аналогию их с результатами исследований на одноступенчатом компрессоре. Максимальный КПД компрессора увеличился на 1,5\%, а оптимальный режим по расходу сместился в сторону меньшего значения. Диапазон расходов, где происходил рост КПД, соответствует режимам повышенной нагруженности венцов группы первых ступеней МОК. Граница газодинамической устойчивости сместилась в сторону меньших расходов на 2,5\%[1].

\section{3 Влияние КК, расположенных над входными кромками рабочих ло- паток МОК, на удельный расход топлива ГТД}

В статор компрессора ГТД после цикла испытаний с серийными рабочими кольцами были установлены доработанные рабочие кольца всех десяти ступеней. В уплотняющем покрытии доработанного рабочего кольца каждого рабочего колеса выполнена кольцевая канавка прямоугольного сечения глубиной, равной толщине покрытия, и шириной, составляющей $20 \%$ осевой проекции хорды концевого сечения рабочей лопатки. Положение каждой канавки задается таковым, чтобы в собранном компрессоре она располагалась над торцами соответствующих рабочих лопаток в зоне их входных кромок.

Указанные выше технические требования соответствуют техническому заданию, разработанному в ХАИ, на базе исследований компрессора с НРУ, Выполненных И.И. Рединым.

Дроссельные характеристики ГТД АИ-24, с серийными и доработанными кольцами показаны на рис. 10.

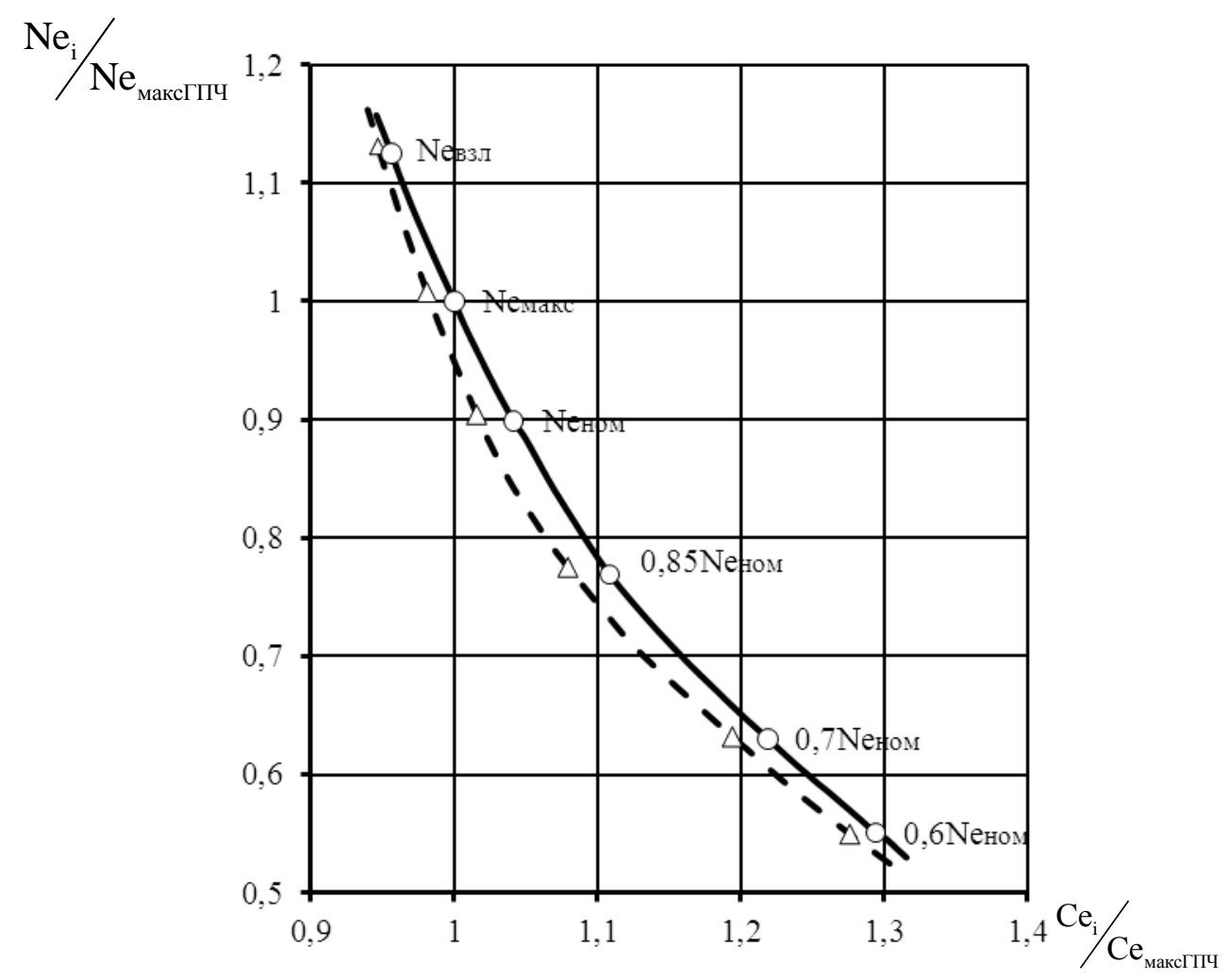

Рис. 10. Дроссельные характеристики ГТД АИ-24:

$$
\begin{aligned}
& \text { - - - стенка ГПЧ; } \\
& \longrightarrow \triangle-\text { - стенка с КК }
\end{aligned}
$$


Была проведена оценка значимости расхождения средних значений удельного расхода топлива Се на каждом из основных режимов ГТД для сравниваемых вариантов изделия: с серийными рабочими кольцами компрессора и опытными (доработанными) рабочими кольцами.

$$
\Delta C e=\frac{\bar{C}_{e \Gamma \Pi \Psi}-\bar{C}_{e K K}}{\bar{C}_{e Г \Pi \Psi}},
$$

где $\bar{C}_{e K K}$ - среднее значение удельного расхода топлива (для каждого из режимов) ГТД с КК;

$\bar{C}_{\text {ГППЧ }}$ - среднее значение удельного расхода топлива (для каждого из режимов) ГТД с ГПЧ.

Разница в удельном расходе топлива по режимам работы ГТД составила:

- максимальный $\Delta \bar{C} e=1,6 \%$;

- номинальный $\Delta \bar{C} e=2,4 \%$;

- 0,85 номинального $\Delta \bar{C} e=2,6 \%$.

Таким образом, на основных режимах работы ГТД АИ-24 получено значимое снижение удельного расхода топлива.

\section{Заключение}

Конструктивно простое технологичное надроторное устройство - кольцевая канавка над входными кромками РК позволяет снизить удельный расход ГТД путем повышения КПД МОК.

Повышение КПД МОК обусловлено положительным влиянием КК на течение в группе первых ступеней, работающих при положительных углах атаки на нерасчетных режимах работы МОК.

\section{Список литературы}

1. Загоруй, В.А. Влияние кольцевых канавок в корпусе компрессора на его характеристики и газодинамическую устойчивость [Текст] / В.А. Загоруй, И.И. Редин // Газовая динамика двигателей и их элементов: сб. науч. тр. Харьков. авиац. ин - та. - 1987. - Вып. 3. - С. $10-15$.

2. Nezym, V. Investigation of design features of compressor casing treatment [Text]/ V. Nezym // The archive of mechanical engineering. - 2014. - T. 61, № 13, C. $153-161$.

3. Ершов, В.Н. Неустойчивые режимы турбомашин [Текст] : монографрия / В.Н. Ершов. - М.: Машиностроение. 1966. - 180 с.

4. Таката, Цукуда. Механизм и эффективность запаса по срыву с помощью перфорирования поверхности корпуса [Текст] / Таката, Цукуда // Тр. амер. о - ва инженеров-механиков. Сер. Энергетические машины и установки. - 1977. - № 1. - C. $134-147$.

5. Грейцер. Явление срыва потока в осевых компрессорах (обзор). [Текст] / Грейцер // Тр. амер. о - ва инженеров-механиков. Сер. Теоретические основы инженерных расчетов. - 1980. - № 2. - С. 72 - 97.

6. Hathaway, M.D. Passive endwall treatments for enhancing stability [Text] / M.D Hathaway // NASA Report. - 2007. - № TM-2007-214409. - 78 p. 
7. Osborn, W.M, Lewis, G.W and Heidelberg, L.J. Effect of several porous casing treatments on stall limit and overall performance of an axial compressor rotor [Text] / W.M. Osborn, G.W. Lewis and L.J. Heidelberg // NASA Report. - 1971. - № TN 6537. $-48 \mathrm{c}$.

8. F04D27/02. Способы и устройства для устранения помпажа [Текст] / Ф.Ш. Гельмедов, Е.А. Локштанов, Л.Е. Ольштейн, М.А. Сидоркин - РФ 2034175; заявл. 1993-03-11; опубл. 30.04.1995.

9. Seitz, P.A. Casing treatment for axial flow compressors [Text] / P.A. Seitz // PhD thesis published at Cambridge University. 1999

10. Noureddine, D. Parametric study for lossless casing treatment on a mixedflow compressor rotor [Text] / D. Noureddine, H. Djeghri, V. Duc // Paper in Proceedings of ASME Turbo Expo 2015: Turbine Technical Conference and Exposition, Montréal, Canada. - 2015, vol. 2A: Turbomachinary. - no. GT2015-42750. - pp. 25-37.

11. Mileshin V. Application of Casing Circumferential Grooves to Counteract the Influence of Tip Clearance [Text] / V. Mileshin, I. Brailko, A. Startsev // ASME Paper. 2008. - № GT2008-51147. - C. 617-627.

12. Ануров, Ю. М. Особенности применения надроторных кольцевых устройств в осевых компрессорных ступенях [Текст] / Ю. М. Ануров, В. А. Коваль, В. Е. Михайлов // Газотурбинные технологии. - 2016. - № 5. - С. 42 - 45.

13. Martin, W. M. Interaction of Rotor and Casing Treatment Flow in an Axial Single-stage Transonic Compressor with Circumferential Grooves [Text] / W. M. Martin, B. Christoph, S. Heinz-Peter, H. Chunill //ASME Paper. - 2008. - №. GT2008-50135. C. 67-78.

14. Prince, D.C. Study of casing treatment stall margin improvement phenomena [Text] / D.C. Prince, D.C. Wisler, D.E. Hilvers // NASA Report. - 1974. - № CR-134552. $-166 \mathrm{p}$.

15. Wilke, I. A numerical investigation of the flow mechanisms in a high-pressure compressor front stage with axial slots [Text] / I. Wilke, H-P. Kau // ASME Journal of Turbomachinery. - 2004. - T. 126(2). - C. 339-349.

16. Fujita, H. A study on configurations of casing treatment for axial flow compressors slots [Text] / Fujita H and Takata H // JSME Bulletin. - 1984. - № 27(230)., C. 1675-1681.

17. Редин, И.И. Диагностика предпомпажного состояния осевого компрессора с надроторным устройством [Текст] / И.И.Редин, М. А. Шевченко // Авиационнокосмическая техника и технология. - 2018. - № 8 (152). - С. 97-107.

18. Houghton, T. Enhancing the stability of subsonic compressors using casing grooves [Text] / T. Houghton, I. Day // ASME Journal of Turbomachinery. - 2009. - № GT2009-59210. - C. 39-48.

19. Shabbir, A. Flow mechanism for stall margin improvement due to circumferential casing grooves on axial compressors [Text] / A. Shabbir, J.J. Adamczyk // ASME Journal of Turbomachinery. - 2005. -T. 127(3), C. 708-717

20. Lakshiminarayana, B. Three-Dimensional Flow Field in the Tip Region of a Compressor Rotor Passage [Text] / B. Lakshiminarayana, M. Pouagare, and R. Davino //Journal of Engineering for Power. - 1982. - T. 104(4). - C. 760-781.

21. Pandya, A. Investigation of the Tip Clearance Flow Inside and at the Exit of a Compressor Rotor Passage [Text] / A. Pandya, B. Lakshminarayana // Journal of Engineering for Power. -1983. - T.105(1). - C. 1-17.

22. Редин, И.И. Влияние щелевого устройства на характеристики и диапазон устойчивых режимов работы рабочего колеса ступени осевого компрессора 
[Текст] / И.И. Редин // Газовая динамика двигателей и их элементов: сб. науч. тр. Харьков. авиац. ин - та. - 1979. - Вып. 1. - С. 65 - 70.

23. Незым, В.Ю. Об опыте применения математической обработки результатов эксперимента при исследовании компрессорного рабочего колеса с высоким КПД [Текст] / В.Ю. Незым // Газовая динамика двигателей и их элементов: сб. науч. тр. Харьков. авиац. ин - та. - 1979. - Вып. 1. - С. 107-109.

24 .Стенд для испытания осевых компрессоров на базе турбовального ГТД [Текст] / А.В. Бусургин, А.И. Егорова, В.Н. Ершов и др. // Энергетическое машиностроение, Харьков. - 1978. - Вып. 25. - С. 85-90.

\section{References}

1. Zagorui, V.A., Redin, I.I. Vliyanie kol'tsevykh kanavok v korpuse kompressora na ego kharakteristiki i gazodinamicheskuyu ustoichivost' [Effect of annular grooves in the compressor casing on its characteristics and gas-dynamic stability]. Gazovaya dinamika dvigatelei i ikh elementov, 1987, pp. $10-15$.

2. Nezym, V. Investigation of design features of compressor casing treatment. The archive of mechanical engineering, 2014, vol. 61, no. 13, pp. $153-161$.

3. Ershov V.N. Neustoichivye rezhimy turbo-mashin [The instability regimes of turbomachinery]. Moscow, Mashinostroenie Publ., 1966. 180 p.

4. Takata, Tsukuda. Mekhanizm i effektivnost' zapasa po sryvu s pomoshch'yu perforirovaniya poverkhnosti korpusa [The mechanism and efficiency stall margine zone via perforating compressors casing surface]. Energeticheskie mashiny i ustanovki: Tr. Amerik. obshch-va inzhenerov-mekhanikov, 1977, vol. 1, pp. $134-147$.

5. Greitser. Yavlenie sryva potoka v osevykh kom-pressorakh (obzor) [The phenomenon of flow stall in axial compressors (review)]. Teoreticheskie osnovy inzhenernykh raschetov: Tr. Amerik. obshch-va inzhener-ov-mekhanikov, 1980, no. 2, pp. $72-97$.

6. M. D. Hathaway. Passive Endwall Treatments for Enhancing Stability. Von Karman Institute for Fluid Dynamics Lecture Series 2006-06: Advances in Axial Compressor Aerodynamics. Von Karman Institute, Rhode St-Genese, Belgium, 2006. Also published as NASA Report, 2007, no. TM-2007-214409, 78 p.

7. Osborn, W.M, Lewis, G.W and Heidelberg, L.J. Effect of several porous casing treatments on stall limit and overall performance of an axial compressor rotor. NASA Report, 1971, no. TN 6537, 48 p.

8. F04D27/02. Sposoby i ustroistva dlya ustraneniya pompazha [Anti-stall tip treatment means] / Gelmedov, F.S, Lokshtanov, E.A, Olstain, L.E.M and Sigorkin, M.A RF 2034175; zayavl. 1993-03-11; opubl. 30.04.1995.

9. P. A. Seitz. Casing Treatment for Axial Flow Compressors. PhD thesis, University of Cambridge, Cambridge, UK, 1999.

10. Noureddine, D., Huu, Duc Vo Parametric study for lossless casing treatment on a mixed-flow compressor rotor. Paper in Proceedings of ASME Turbo Expo 2015: Turbine Technical Conference and Exposition, Montréal, Canada, 2015, vol. 2A: Turbomachinary, no. GT2015-42750, pp. 25-37.

11. Victor Mileshin, Igor Brailko and Andrew Startsev Application of Casing Circumferential Grooves to Counteract the Influence of Tip Clearance. ASME Paper, 2008, no. GT2008-51147, pp. 617-627. 
12. Anurov Y. M., Koval V. A., Mikhailov V. E. Osobennosti primeneniya nadrotornykh kol'tsevykh ustroistv $v$ osevykh kompressornykh stupe [Forecasting stall modes of axial compressors gte taking into account features of formation of profile and end boudary layers]. Gazoturbinnye tekhnologii, 2016, no. 5, pp. $42-45$.

13. Martin W. Muller, Christoph Biela, Heinz-Peter Schiffer, Chunill Hah Interaction of Rotor and Casing Treatment Flow in an Axial Single-stage Transonic Compressor with Circumferential Grooves. ASME Paper, 2008, no. GT2008-50135, pp. 67-78.

14. Prince D.C, Wisler D.C and Hilvers D.E. Study of casing treatment stall margin improvement phenomena. NASA Report, 1974, no. CR-134552, 166 p.

15. Wilke I and Kau H-P. A numerical investigation of the flow mechanisms in a high-pressure compressor front stage with axial slots. ASME J. Turbomachinery, 2004, vol. 126(2), pp. 339-349.

16. Fujita $\mathrm{H}$ and Takata $\mathrm{H}$. A study on configurations of casing treatment for axial flow compressors. JSME Bulletin, 1984, vol. 27(230), pp. 1675-1681.

17. Redin, I.I., Shevchenko, M.A. Diagnostika predpompazhnogo sostoyaniya osevogo kompressora s nadrotornym ustroistvom [Diagnostics of pre-surge condition of the axial compressor with the annular grooves casing treatment]. Aviatsionnokosmicheskaya tekhnika i tekhnologiya, 2018, no. 8 (152), pp. 97-107.

18. Houghton, T., Day, J. Enhancing the stability of subsonic compressors using casing grooves. ASME Journal of Turbomachinery, 2009, no. GT2009-59210, pp. 3948.

19. Shabbir, A., Adamczyk, J. J. Flow Mechanism for Stall Margin Improvement due to Circumferential Casing Grooves on Axial Compressors. ASME Journal of Turbomachinery, 2005, vol. 127(3), pp. 708-717.

20. Lakshiminarayana, B., Pouagare, M., Davino, R. Three-Dimensional Flow Field in the Tip Region of a Compressor Rotor Passage. ASME Journal of Engineering for Power, 1982, vol. 104(4), pp. 760-781,

21. Pandya A., Lakshminarayana, B. Investigation of the Tip Clearance Flow Inside and at the Exit of a Compressor Rotor Passage /ASME Journal of Engineering for Power, 1983, vol. 105(1), pp. 1-17.

22. Redin. I.I. Vliyanie shchelevogo ustroistva na kharakteristiki i diapazon ustoichivykh rezhimov raboty rabochego kolesa stupeni osevogo kompressora [The effect of slit device to the characteristics and range of operation stable modes of stage axial compressor]. Gazovaya dinamika dvigatelei i ikh elementov, 1979, vol. 1, pp. $65-$ 70.

23. Nezym, V.Yu. Ob opyte primeneniya matematicheskoi obrabotki rezul'tatov eksperimenta pri issledovanii kompressornogo rabochego kolesa s vysokim KPD [About the experience of applying mathematical processing of experimental results in the study of a compressor rotor with high efficiency]. Gazovaya dinamika dvigatelei i ikh elementov, 1979, vol. 1, pp. $107-109$.

24. Busurgin, A.V., Egorova, A.I., Ershov V.N. Stend dlya ispytaniya osevykh kom-pressorov na baze turboval'nogo GTD [Stand for testing of axial compressors based on turboshaft engine]. Energeticheskoe mashinostroenie, Khar'kov, 1978. - vol. 25. - pp. 85-90. 


\section{Покращення паливної ефективності газотурбінного двигуна встановленням у компресорі надроторного пристрою}

Наведено аналіз впливу кільцевих канавок на течію в проточній частині робочого колеса і характеристики осьового компресора, а також на паливну ефективність газотурбінного двигуна в цілому. Викладено можливий механізм впливу течії в порожнині кільцевої канавки на основну течію в кінцевій області проточної частини робочого колеса ступеня осьового компресора. Ефективність надроторного пристрою у вигляді однієї кільцевої канавки, ширина якої становить $20 \%$ осьової проекції хорди кінцевого перерізу лопатки робочого колеса, показано експериментально в системі одноступінчастого і багатоступінчастого осьового компресора. Зростання ККД компресора з десятьма одиночними кільцевими канавками, встановленими над вхідними кромками лопаток робочих коліс кожного ступеня, забезпечив зниження питомої витрати палива серійного ГТД на основних режимах його роботи.

Ключові слова: надроторний пристрій, паливна ефективність, течія в радіальному зазорі, газодинамічна стійкість, осьовий компресор.

\section{Improving the fuel efficiency of a gas turbine engine by installing in the compressor a casing treatment}

The analysis of the influence of annular grooves on the flow in the compressor rotor airgas channel and the axial compressor characteristics as well as on the fuel efficiency of the gas turbine engine is presented. The hypothetical mechanism of the flow effect in the cavity of the annular groove on the main flow in the tip end of the blade airfoil of the axial compressor stage is outlined. The effectiveness of the casing treatment in the form of single annular groove, width is $20 \%$ of the axial projection of the chord of the tip end section of the blade is shown experimentally in a single-stage and multistage axial compressor system. The increase of the compressor efficiency with ten single annular grooves installed above the leading edges of the blades of each stage, has reduced the specific fuel consumption of the serial GTE in its main operating modes.

Key words: annular groove casing treatment, fuel efficiency, radial clearance flow, gas - dynamic stability, axial compressor.

\section{Сведения об авторах:}

Редин Иван Иванович - доцент кафедры теории авиационных двигателей Национального аэрокосмического университета им. Н. Е. Жуковского «Харьковский авиационный институт», Харьков, Украина, e-mail: i.redin@khai.edu.

Шевченко Михаил Анатольевич - аспирант кафедры теории авиационных двигателей Национального аэрокосмического университет им. Н. Е. Жуковского «Харьковский авиационный институт», Харьков, Украина, e-mail: mikleshevchenko@gmail.com, ORCID: 0000-0002-0806-6632.

Redin Ivan Ivanovich - Assistant Professor at the department of Aviation Engines Theory, National Aerospace University named after N. Ye. Zhukovsky "Kharkiv Aviation Institute", Kharkiv, Ukraine, e-mail: i.redin@khai.edu.

Shevchenko Mikhail Anatol'evich - PhD student at the department of Aviation Engines Theory, National Aerospace University named after N. Ye. Zhukovsky "Kharkiv Aviation Institute", Kharkiv, Ukraine, e-mail: mikleshevchenko@gmail.com, ORCID: 0000-0002-0806-6632. 